\title{
ReaR
}

ISNN 19894090

Revista electrónica de AnestesiaR

LECTURA CRÍTICA DE ARTÍCULO

Mayo 2018

\section{Recomendaciones ERS/ATS para el uso de la ventilación no invasiva en la insuficiencia respiratoria aguda. ¿Hay novedades?}

Artículo original: Bochwerg B, Brochard L, Elliott MW, Hess D, Hill NS, Nava S et al. Official ERS/ATS clinical practice guidelines: noninvasive ventilation for acute respiratory failure. Eur Respir J 2017; 50: 1602426 (Pubmed) (HTML) (PDF)

Jiménez Vizuete JM, Iñiguez De Onzoño A, Sánchez López.

Hospital General Universitario de Albacete.

\section{Resumen}

La insuficiencia respiratoria aguda (IRA) sigue siendo una patología frecuente en la Unidad de Cuidados Intensivos (UCI). Van a ser múltiples las causas de la IRA, desde descompensaciones de patologías respiratorias crónicas, contusiones pulmonares por traumatismos, postoperatorios de cirugía de resección pulmonar, sepsis de origen respiratorio por neumonías comunitarias o asociadas a cuidados sanitarios o bien por el desarrollo de un síndrome de distrés respiratorio agudo (SDRA) asociado a otras patologías extrapulmonares. El tratamiento de la IRA es el de la causa que lo provoca, siendo la ventilación mecánica el soporte necesario para mantener al paciente oxigenado mientras se curan los daños pulmonares causantes de la misma. El soporte respiratorio puede variar desde la simple aplicación de oxigenoterapia, hasta la intubación orotraqueal y conexión a la ventilación mecánica invasiva (VMI), pasando por la ventilación no invasiva (VNI) en diferentes modalidades. Además hay que tener en cuenta que la VNI puede ayudar a evitar intubaciones o facilitar la extubación de determinados pacientes.

\section{Introducción}

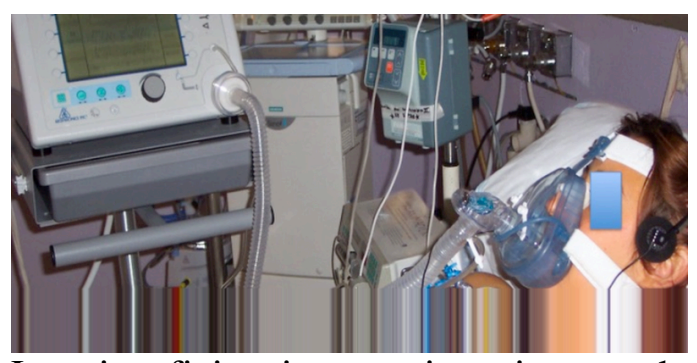

La insuficiencia respiratoria aguda (IRA) sigue siendo una patología frecuente en la Unidad de Cuidados Intensivos (UCI). Van a ser múltiples las causas de la IRA, desde descompensaciones de patologías respiratorias crónicas, contusiones pulmonares por traumatismos, postoperatorios de cirugía de resección pulmonar, sepsis de origen respiratorio por neumonías comunitarias o asociadas a cuidados sanitarios o bien por el desarrollo de un síndrome de distrés respiratorio agudo (SDRA) asociado a otras patologías extrapulmonares ${ }^{1}$. El tratamiento de la IRA es el de la causa que lo provoca, siendo la ventilación mecánica el soporte necesario para mantener al paciente oxigenado mientras se curan los daños pulmonares causantes de la misma. El soporte respiratorio puede variar desde la simple aplicación de oxigenoterapia, hasta la intubación orotraqueal y conexión a la ventilación mecánica invasiva (VMI), pasando por la ventilación no invasiva (VNI) en diferentes modalidades. Además hay que tener en cuenta que la VNI puede ayudar a evitar intubaciones o facilitar la extubación de determinados pacientes. 


\section{Resumen}

\section{Objetivos}

Este trabajo pretende dar unas recomendaciones, basadas en la evidencia científica, sobre el uso clínico de la VNI en la IRA y aspectos prácticos relacionados con la aplicación y mantenimiento de la misma. En este documento, la VNI incluye la presión positiva variable en la vía aérea (Bilevel), así como la presión positiva continua en la vía aérea (CPAP).

\section{Material y Métodos}

La metodología y formulación de recomendaciones, se llevó a cabo por miembros de un comité de expertos en el campo de la VNI, miembros de la European Respiratory Society y de la American Thoracic Society. Este comité estaba compuesto por médicos (neumólogos o intensivistas) $\mathrm{y}$ terapeutas respiratorios, además de dos clínicos con experiencia en medicina basada en la evidencia.

Este comité desarrolló unas directrices para 11 preguntas basadas en la importancia clínica, usando el formato PICO (población - intervención comparación - resultado). Se realizó una búsqueda de la literatura, selección de estudios potenciales, agrupación de datos y un resumen y análisis de la evidencia para su selección definitiva.

Para la clasificación de la calidad de la evidencia y fuerza de las recomendaciones formuladas en este trabajo se siguió la propuesta del grupo GRADE (Grading of Recommendations Assessment, Development and Evaluation Working Group). La búsqueda bibliográfica se realizó usando la plataforma OVID buscando en la base de datos MEDLINE / PubMed y Registro Cochrane de ensayos controlados. La última actualización de la búsqueda se realizó en noviembre de 2016. Se limitó la búsqueda a "humanos" e idioma "inglés" o cualquier idioma con resúmenes en inglés.

El comité seleccionó los resultados de interés para cada pregunta y éstos fueron clasificados en función de su importancia de "no importante" a "crítico". Los resultados prioritarios de los estudios analizados fueron: mortalidad, necesidad de intubación y tratamiento de la neumonía nosocomial, considerando la disnea notificada por el paciente, un resultado crítico.

\section{Resultados}

Un resumen de las recomendaciones traducidas de este trabajo se presenta en la tabla 1.
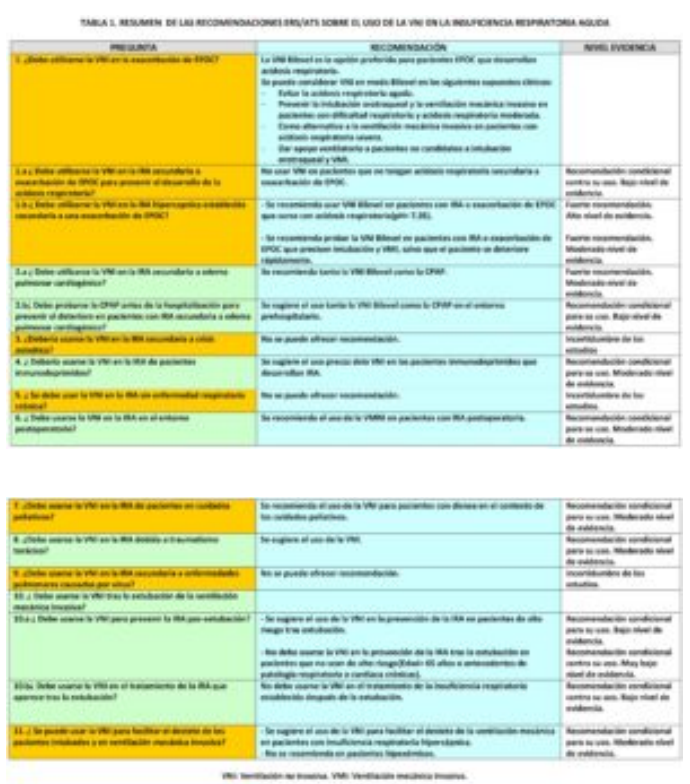

Tabla 1. Resumen de las recomendaciones ERS/ATS sobre el uso de la VNI en la insuficiencia respiratoria aguda.

\section{Conclusiones}

Atendiendo a la revisión de la literatura, son diversos los usos clínicos que se le puede dar a la VNI. Se deberá esperar a futuros estudios que comparen la VNI con nuevas terapias como las gafas nasales de altos flujos o terapia 
extracorpórea de eliminación de $\mathrm{CO} 2$. La utilización de la VNI debe hacerse, aplicando el buen juicio clínico, en función de las características del paciente y la experiencia personal.

\section{Comentarios}

Se puede definir la VNI como cualquier forma de soporte ventilatorio aplicado sin el uso de un tubo orotraqueal. Un aspecto clave de la VNI es la posibilidad de evitar la intubación traqueal y la VMI y sus potenciales complicaciones. Además, es más confortable, el paciente puede comunicarse, comer y beber, expectorar, evita la necesidad de sedación profunda y se preservan los mecanismos de defensa de la vía aérea superior. Los objetivos principales de la VNI en la IRA son reducir el trabajo respiratorio y corregir la hipoxemia y la acidosis respiratoria. Estos objetivos pueden variar en función del tipo de insuficiencia respiratoria, del contexto clínico y de la enfermedad de base del paciente $^{2}$.

La VNI es actualmente el tratamiento de elección en la mayor parte de pacientes que desarrollan IRA. Los buenos resultados obtenidos en las agudizaciones graves de la EPOC han extendido el uso de la VNI a otras patologías que cursan con fallo respiratorio agudo, siendo hoy considerada una intervención de primera línea en el manejo de muchos de estos pacientes. El éxito de la VNI depende en gran medida de una selección adecuada de los pacientes. Para ello, deben tenerse en cuenta las características clínicas del enfermo, la causa y potencial reversibilidad de la insuficiencia respiratoria y el riesgo de fracaso de la $\mathrm{VNI}^{3}$.

Esta guía pretende actualizar la evidencia científica del uso de la VNI en diferentes situaciones clínicas, que se pueden presentar en la práctica diaria y sobre las cuales queremos hacer algunos comentarios.

A diferencia de la VMI convencional la VNI ofrece la oportunidad de aportar asistencia ventilatoria fuera de unidades de cuidados intensivos, ya que se puede aplicar de forma intermitente, no requiere sedación y permite además una intervención en fases iniciales de la insuficiencia respiratoria $y$ en un entorno menos hostil para el paciente. Asimismo, puede reducir los elevados costes que supone la atención en UCI. La presencia de unidades específicas de cuidados respiratorios o unidades de cuidados intermedios, puede aliviar la carga de la UCI al permitir el tratamiento de pacientes con deterioro respiratorio que se pueden manejar con VNI. Como en otros aspectos de la sanidad, son los recursos económicos disponibles, los que limitan la presencia de este tipo de unidades en muchos de nuestros hospitales.

La exacerbación de la EPOC es la patología más estudiada y la que más se puede beneficiar del uso de la VNI, siendo la presencia de la acidosis respiratoria, la que presenta un mayor nivel de evidencia para el uso de la misma. Respecto a probar la VNI en la exacerbación de la EPOC que requiere intubación orotraqueal, creemos que debemos seleccionar muy bien al paciente para evitar un deterioro crítico del mismo. Pensamos que en este caso, un buen nivel de consciencia nos puede hacer probar la VNI antes de intubarlo, en caso contrario proceder a intubar directamente.

Hay nuevas indicaciones que nos parecen interesantes. Por un lado, el uso prehospitalario, para prevenir el deterioro el paciente con IRA secundaria a edema pulmonar cardiogénico, aunque esto implica disponer de buenos respiradores en el 
entorno prehospitalario y la formación correcta de los profesionales. Otro caso es el uso en medicina paliativa para evitar la disnea de los pacientes. En este caso además de disponer de respiradores y formación pensamos que hay que explicar muy bien al paciente y a familiares el por qué se le aplica la VNI y evitar prolongar la agonía de los pacientes. Por último, en pacientes que han expresado su deseo de no ser intubados $o$ en los que no son candidatos, por condiciones médicas a la intubación, la VNI puede plantearse como soporte ventilatorio, en un contexto de adecuación del esfuerzo terapéutico, mientras se resuelve un proceso agudo o como tratamiento paliativo para aliviar la disnea o prolongar brevemente la vida mientras se toman decisiones o se resuelven otros problemas ${ }^{4}$.

Respecto al uso de la VNI en la crisis asmática grave, pensamos que la necesidad de aplicar altos niveles de presión puede resultar muy incómodo para el paciente, no ser bien tolerados y complicar la aplicación del tratamiento con los broncodilatadores inhalados, que se deberían aplicar con una mezcla oxígeno/heliox.

Es habitual usar la VNI para facilitar el destete de la VMI o para prevenir la IRA tras la extubación en los pacientes ingresados en UCI. Estos usos sólo se recomiendan, con bajo nivel de evidencia, en pacientes de alto riesgo de desarrollo de IRA. Llama la atención que no se recomiende el uso de la VNI para tratar la IRA establecida tras la extubación, ya que no disminuye la tasa de reintubación y la mortalidad, comparada con la terapia convencional con oxígeno. Pero la realidad es que se usa a diario y la impresión clínica es que se evita la reintubación en muchas ocasiones.
En los traumatismos torácicos, que cursan con fracturas costales, la VNI junto con la analgesia epidural y la fisioterapia respiratoria son los pilares básicos del tratamiento, evitando en la mayoría de los casos la necesidad de intubación orotraqueal ${ }^{5}$.

Respecto al uso de la VNI en IRA sin patología respiratoria crónica, como un SDRA, si bien no establecen recomendaciones, en un estudio reciente el uso de la VNI se asoció a un aumento de la mortalidad, sobre todo en pacientes con $\mathrm{P} 02 / \mathrm{Fi} 02<150^{6}$.

En estas guías no se entra a valorar la aplicación de las distintas interfaces, ni el tipo de respirador para VNI. Afortunadamente los nuevos respiradores de VMI disponen de modos de VNI que permiten tratar adecuadamente a los pacientes, no siendo necesario disponer de respiradores específicos para VNI.

No hacen referencia las guías a estudios comparativos entre VNI o aplicación de oxígeno en altos flujos mediante gafas nasales, modalidad de tratamiento cada vez más utilizada. En un meta-análisis reciente la aplicación de oxígeno en gafas nasales de altos flujos, parece que es tan eficaz como la VNI, en la reducción de la necesidad de intubación orotraqueal o la reintubación tras la $\mathrm{VMI}^{7}$.

\section{Bibliografía}

1. Vincent JL, Akça S, De Mendoça A, HajiMichael P, Sprung C, Moreno R et al. The epidemiology of acute respiratory failure in critically ill patients. Chest 2002, 121:1602-9. (PubMed) (HTML) (PDF)

2. Mehta S, Hill NS. Noninvasive ventilation. Am J Respir Crit Care Med 2001; 163:540-577. (PubMed) (HTML) (ePDF)

3. Nava S, Ceriana P. Causes of failure of noninvasive mechanical ventilation. Respir Care 2004; 49: 295- 303. (PubMed) (PDF)

4. Curtis JR, Cook DJ, Sinuff T, White DB, Hill N, Keenan SP et al. Noninvasive positive pressure ventilation in critical and palliative care settings: 
understanding the goals of therapy. Crit Care Med 2007; 35: 932-9. (PubMed)

5. Galvagno SN Jr, Smith CE, Varon AJ, Hasenboehler EA, Sultan S, Shaefer G et al. Pain management for

blunt thoracic trauma: A joint practice management guideline from the Eastern Association for the Surgery

of Trauma and Trauma Anesthesiology Society. J Trauma Acute Care Surg 2016;81:936-951.

6. Bellani G, Laffey JG, Pham T, Madotto F, Fan $E$, Bronchard L et al. Noninvasive ventilation of patients with acute respiratory distress syndrome. Insights from the LUNG SAFE study. Am J Respir Crit Care Med 2017;195:6777. (PubMed) (HTML)

7. Ni YN, Luo J, Yu H, Liu D, Liang BM, Liang ZA. The effect of high-flow nasal cannula in reducing the mortality anh the rate of endotracheal intubation when used before mechanical ventilation compred with conventional oxygen therapy and noninvsive positive pressure ventilation. A systematic review and meta-analysis. Am J Emerg Med. 2017; pii: S0735-6757(17)30629-0.doi: 10.1016/j.ajem.2017.07.083. (PubMed)
Correspondencia al autor

José María Jiménez Vizuete

josemvizuete@gmail.com

Jefe de Sección UCI Anestesia. Servicio Anestesiología y Reanimación.

Hospital General Universitario de Albacete.

Aceptado para blog en octubre de 2017. 